\title{
论文
}

\section{一种用于柱面压缩实验并设有两级内翅式换热器 冷屏的液氦温区低温靶}

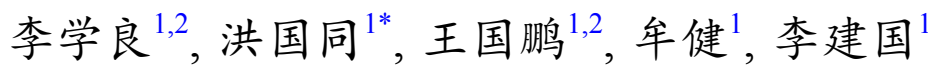 \\ 1. 中国科学院理化技术研究所, 空间功热转换技术重点实验室, 北京 100190 ; \\ 2. 中国科学院大学, 北京 100049 \\ * E-mail: gthong@mail.ipc.ac.cn \\ 收稿日期: 2017-12-19; 接受日期: 2018-05-28; 网络版发表日期: 2018-07-30 \\ 国家重点基础研究发展计划(编号: 613322)资助项目
}

\begin{abstract}
摘要通过低温技术实现物质的高密度状态并进行冲击压缩实验是获得物质高能量密度状态方程的重要途径. 本文介绍了一种用于柱面压缩实验的设有两级内翅式换热器冷屏的液氦温区低温靶. 该低温靶通过设置两级内 翅式换热器冷屏和在低温下可脱落的折叠冷屏结构, 实现了液氦的高效存储, 成功研制了应用于柱面压缩实验的 液氦温区低温靶. 在停止外界液氦供给和关闭真空原的条件下, 可获得 $4.77 \mathrm{~K}$ 的样品室稳定温度, 样品室的降温时 间和维持稳定时间均满足柱面压缩实验的要求。本文还介绍了低温靶的结构设计、利用ANSYS Steady-Static Thermal模块对低温靶进行的热分析与实验结果. 仿真计算结果与实验结果吻合较好.
\end{abstract}

关键词液氦低温靶, 内翅式换热器冷屏, 折叠冷屏, 数值计算

\section{1 引言}

物质处于高能量密度状态时，由于受到外界能量 输入或自身能量转换，其内能增大会造成压力、密度 和温度大幅度提高，相应的状态方程会发生明显变

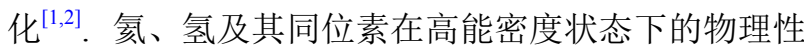
质是天体物理、激光聚变和核物理等学科研究的基础 问题，对于模拟研究星体特性和设计惯性约束核聚变 有重要意义 ${ }^{[3,4]}$. 目前, 高能量密度状态下的物理性质 主要采取冲击压缩技术对相应样品进行加载来获得. 根据Rankine-Hugoniot关系 ${ }^{[5]}$ ，当初始密度提高时，其
冲击压力也会增大．低温靶正是利用低温技术使样品 气在指定的空间(样品室)获得较高的初始密度的液态 或固态样品,进而增大碰撞后的冲击压力, 使其达到高 能量密度状态.

低温靶冲击压缩方式主要有轴向加载和柱面加载 两种形式. 轴向加载主要利用轻气炮冲击技术, 其加载 压力最大可达几十 GPa. 柱面加载则使用内爆磁压缩 技术，是唯一可以使冲击压力达到 $\mathrm{TPa}$ 量级的压缩方 式，更容易满足超高压力的加载需求，已成为主流加 载方式 ${ }^{[6,7]}$. 鉴于液氦、液氢及其同位素冲击压缩实验 的重要性, 国外自20世纪60年代就开展了相关实验, 并

引用格式: 李学良, 洪国同, 王国鹏, 等. 一种用于柱面压缩实验并设有两级内翅式换热器冷屏的液氦温区低温靶. 中国科学: 技术科学, 2018, 48: 827-835 Li X L, Hong G T, Wang G P, et al. A liquid helium temperature target with two-stage internally finned cold shield heat exchanger for cylinder compression experiment (in Chinese). Sci Sin Tech, 2018, 48: 827-835, doi: 10.1360/N092017-00359 
得到了许多有价值的研究成果. 如美国劳伦斯实验 室、俄罗斯实物院等机构均成功研制出用于内爆磁压 缩实验的柱面压缩形式低温靶, 实现了液态 $\mathrm{H}_{2}$ 和 $\mathrm{D}_{2}$ 的 冲击压缩实验 ${ }^{[8,9]}$. 这种柱面压缩低温靶是利用低温液 体(液氮或液氦)作为制冷剂，实验前将制冷剂存储在 低温容器内, 利用制冷剂液体或蒸汽冷却样品室. 该 类型低温靶虽然能将样品室冷却至目标温度, 但冷却 样品室之后的制冷剂直接排向大气, 系统绝热效率低, 制冷剂消耗量较大, 而且大体积的低温容器在加载实 验过程中容易存留较多制冷剂, 在加载过程中存在安 全隐患并影响实验结果. 国内针对低温液体开展冲击 压缩实验的研究较晚, 2013年以前柱面压缩形式的低 温靶技术一直未得到突破, 直到中国科学院理化技术 研究所于 2013年在国内首次研制了柱面压缩液氮温区 低温靶 ${ }^{[10]}$. 该低温靶可通过在容积仅为 $150 \mathrm{~mL}$ 的储液 槽内存储液氮, 实现在断开外界液氮供应后, 样品室内 样品仍能在 $60 \mathrm{~min}$ 内维持稳定的要求. 由于单位体积 液氦的蒸发潜热比液氮小两个量级, 因此, 该液氮温 区低温靶不能直接用做液氦温区低温靶. 目前, 国内 还没有研制出应用于柱面压缩实验的液氦温区低温 靶, 鉴于柱面压缩加载形式的重要意义, 该类型低温靶 的研究工作迫在眉睫.

本文介绍了一种应用于柱面压缩实验的液氦温区 低温靶, 该低温靶利用低温液体氦作为冷源, 对样品室 进行冷却, 实现样品气的液化. 通过在冷氦气排出管路 内安装两级内翅式换热器回收冷氦气显热, 用于冷却 储液槽和样品室的辐射屏(冷屏), 既减少储液槽排气 管路传导漏热, 又可通过冷屏减少进入储液槽的辐射 漏热, 实现在较小储液容积内高效存储液氦的目的. 为使样品室在冲击压缩瞬间裸露于室温加载筒体内, 设计了电机驱动折叠冷屏脱离装置, 可在真空低温环 境下实现折叠冷屏的自动脱落. 采用不同材料和结构 的传冷座设计, 可以在不增加热量加入的基础上实现 不同样品室温度的调节与控制以液化不同样品气.

\section{2 低温靶结构设计}

为了在有限容积内较长时间存储液氦, 以达到在 断开外界液氦供给后, 样品室仍能较长时间维持稳定 温度的目的, 在冷氦气排放管道的内部设置换热器, 换热器为狭缝内翅式, 将该换热器与冷屏连接, 就可
以大大提高冷量回收效率, 实现液氦的高效存储.

图1为本文研制的液氦温区低温靶结构示意图, 液 氦温区低温靶主要由进液组件、储液槽、样品气充气 管、两级内翅式换热器、样品室阀组件、两级固定冷 屏及其下方的折叠冷屏、折叠冷屏驱动组件等组成. 其中阀组件由阀杆调节螺帽、阀杆、弹簧调节环、弹 簧、波纹管、阀球头和温度计组成, 可以在低温下通 过调节螺帽实现阀杆的上下移动, 以便密封样品室腔 体. 折叠冷屏驱动组件由电机、凯夫拉线和冷屏卡子 组成, 降温过程中, 折叠冷屏通过冷屏卡子固定在各 自的冷屏下端, 当进行柱面加载实验时, 可通过外置 电机拉紧凯夫拉线, 使得与之相连的冷屏卡子脱开, 折叠冷屏脱落, 折叠到加载模拟器下部, 样品室裸露 至室温加载筒体内.

储液槽为 $220 \mathrm{~mL}$ 的圆筒形腔体结构, 液氦通过进 液管路注入到储液槽内, 在断开外界液氦供给后, 由 于系统的漏热, 液氦会不断蒸发, 蒸发的冷氦气沿气 体管路排出. 蒸发出来的低温冷氦气具有较大的显热 (或显冷), 当冷氦气流过换热器翅片时, 其显热被部分 回收, 用于冷却换热器和与之相连的冷屏, 进而减少储 液槽在排气管路的传导漏热和加载筒体对储液槽的辐 射漏热, 提高液氦的存储时间.

图2为内翅式换热器冷屏结构图, 图3为换热器在 排气管路中的安装示意图, 换热器内侧设计了50个沿 周向均匀分布的长条狭缝, 狭缝宽度 $0.4 \mathrm{~mm}$, 长度 $4.5 \mathrm{~mm}$, 换热器高度 $37 \mathrm{~mm}$, 材料选用导热较好的紫 铜, 中心孔安装杜瓦内管, 杜瓦内管内安装阀杆. 蒸发 的冷气体只能由换热器狭缝内流过, 保证充分换热.

样品室为内径 $3.4 \mathrm{~mm}$, 外径 $6 \mathrm{~mm}$, 深 $53 \mathrm{~mm}$ 的柱 形空腔. 样品室入口设有微型球头密封阀, 在样品气 进入样品室液化并注满后, 通过阀杆组件的阀球头向 下移动关闭该低温阀, 使样品室形成一个密闭空间, 封存液化的样品. 阀门球头内部安装了二极管温度计, 通过其温度的变化监测样品液面的位置.

为了减少辐射漏热, 在储液罐和样品室外部均设 置防辐射冷屏. 考虑到压缩实验过程样品室周围不能 有遮挡, 为此将样品室外部的冷屏设计成可自由脱落 的折叠结构. 折叠冷屏由卡子固定在储液罐冷屏的下 部, 储液罐冷屏的上部与冷屏换热器相连. 在样品室 注满液化的样品进行压缩实验前, 通过外部电机驱动, 使折叠冷屏上部的卡子脱开, 折叠冷屏在真空低温环 
剖图A-A
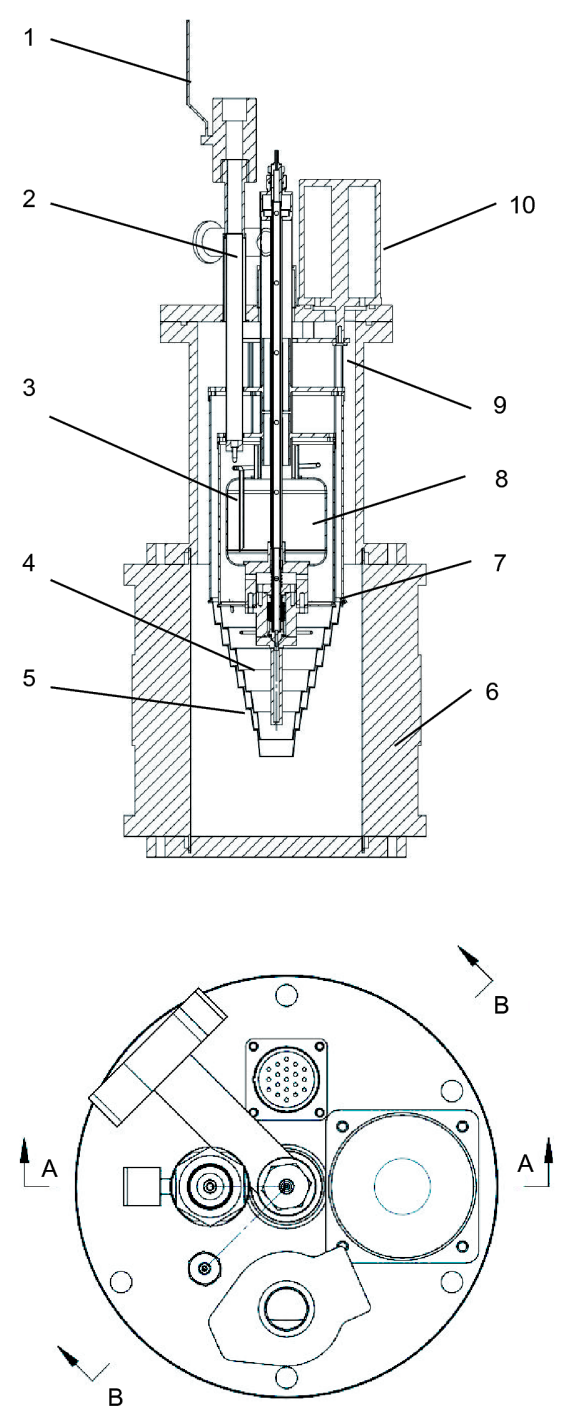

剖图B-B
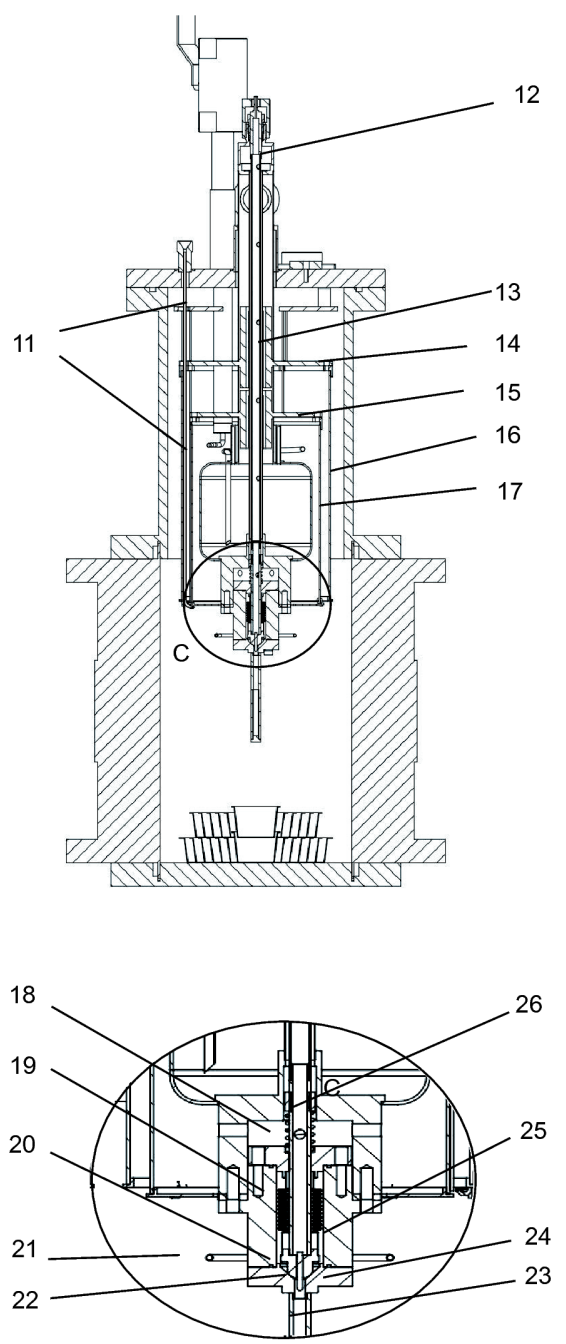

图 1 液氦温区低温靶结构示意图. 1 球阀; 2 进液直管; 3 进液螺旋管; 4 第一级折叠冷屏; 5 第二级折叠冷屏; 6 柱面加载模拟 器; 7 冷屏卡子; 8 储液槽; 9 凯夫拉线; 10 外置电机; 11 充气直管; 12 阀组件; 13 杜瓦内管; 14 第二级内翅式换热器; 15 第一级 内翅式换热器; 16 第二级圆筒形冷屏; 17 第一级圆筒形冷屏; 18 弹簧; 19 波纹管; 20 阀球头; 21 充气弯管; 22 温度计; 23 样品 室; 24 阀座; 25 传冷座; 26 弹簧调节环

境下从储液罐冷屏上脱落后落在真空腔体的下部并折 叠(如图1的B-B视图), 样品室完全暴露于室温加载腔 体内筒体.

此时应立即完成柱面压缩实验，防止样品室液体 发生大量气化, 图4为折叠冷屏结构示意图.

为得到不同样品室温度，以液化不同沸点的样品 气，同时不带来附加热量，保证储液槽保温时间，通过 更换具有不同结构尺寸和材料的传冷座来调节储液槽
和样品室之间的温差, 结合微加热控温措施, 使得样品 室可以维持在 $5 \sim 14 \mathrm{~K}$ 之间任一稳定温度.

低温靶主体置于真空腔体内, 当真空度达到 $10^{-2} \mathrm{~Pa}$ 以上时低温储液槽和样品室的对流漏热可以忽 略不计, 只有少量的残余气体导热. 靶体内管路均使用 壁厚为 $0.2 \sim 0.5 \mathrm{~mm}$ 的薄壁不锈钢管，通过减小导热面 积、增加长度来减少传导漏热. 所有冷屏表面均镀金 处理, 并对储液槽、外冷屏、排气管等低温表面包扎 


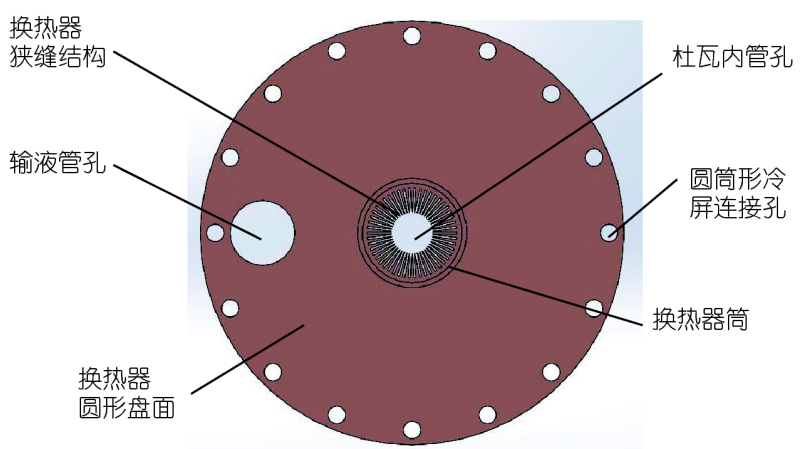

图 2 (网络版彩图)换热器结构示意图

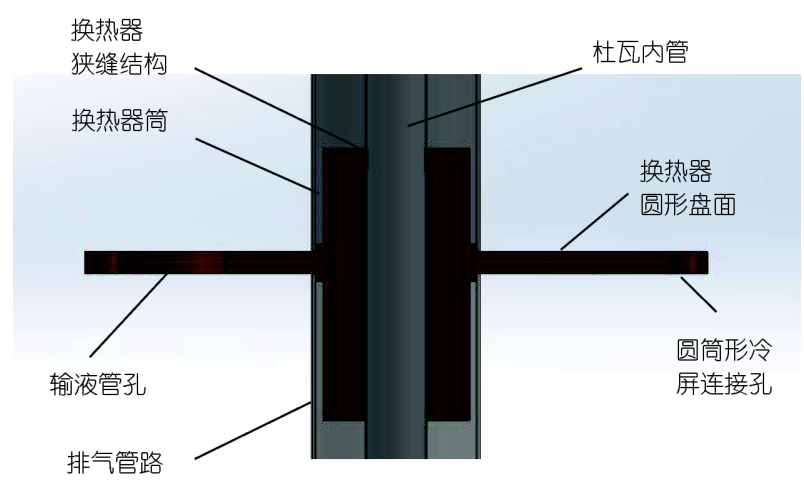

图 3 (网络版彩图)换热器安装示意图
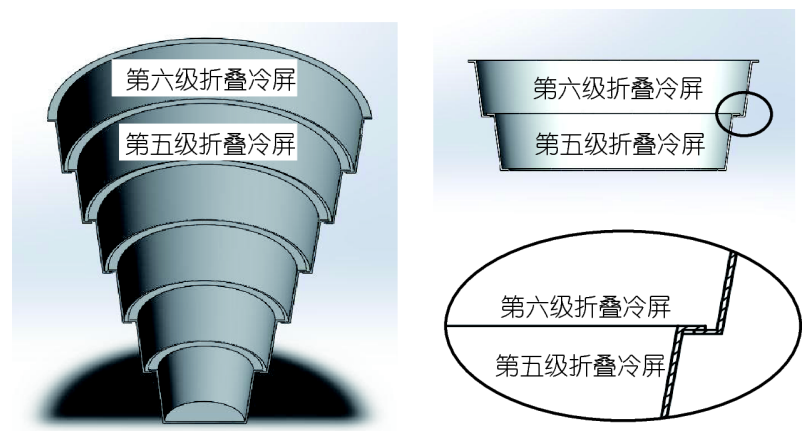

图 4 折叠冷屏结构示意图

多层绝热材料, 以减少辐射漏热.

\section{3 数值计算}

针对低温靶建立如图5所示的传热模型. 模型中, 各热阻以 $R$ 表示, 即导热热阻以 $R_{\lambda}$ 表示, 辐射热阻以 $R_{f}$ 表示; $q_{\lambda}$ 和 $q_{f}$ 分别表示导热热流和辐射热流; 假设两级 换热器和与之相连接的圆筒形冷屏温度一致，分别以 $T_{1}$ 和 $T_{2}$ 表示; 在传热计算过程中, 可先将折叠冷屏的温

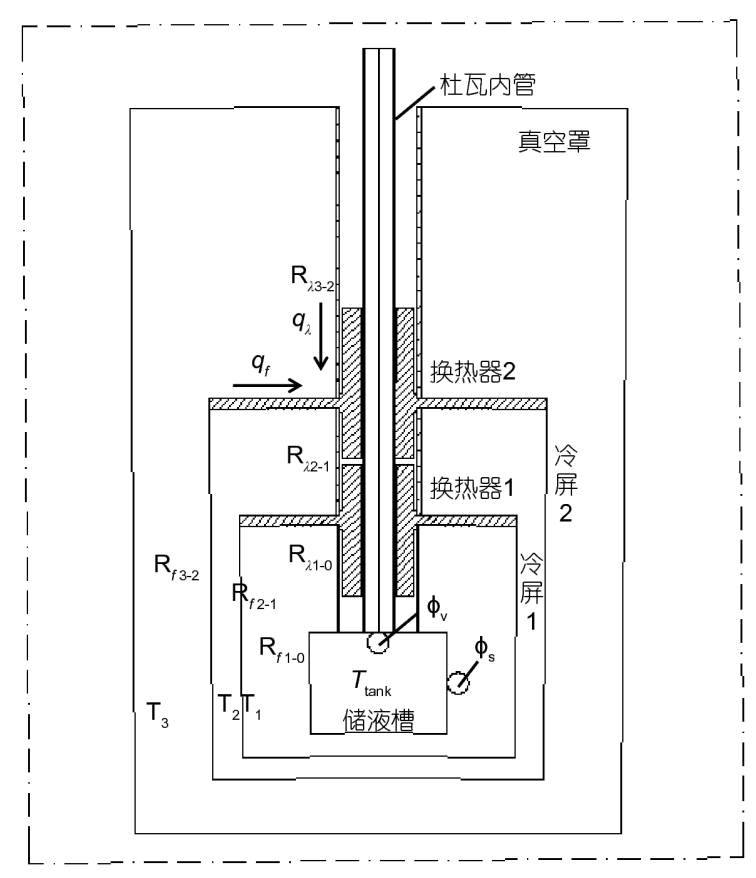

图 5 低温靶传热模型

度视为一致并按照与之相连的圆筒形冷屏的温度进行 计算，各级折叠冷屏的温度可在得到迭代计算换热器 温度后，在ANSYS Steady-Static Thermal模块设置相 应接触热阻模拟计算得到.

对储液槽而言，传热路径包括第一级狭缝换热器 冷屏对其辐射漏热 $\Phi_{f 1-0}$ 、颈管传导漏热 $\Phi_{\lambda 1-0}$ 、杜瓦内 管传导漏热 $\Phi_{\lambda c 1-0}$ 、输液管传导漏热 $\Phi_{\lambda p}$ 、阀组件传导 漏热 $\Phi_{\lambda v}$ 和充气液化管路传导漏热 $\Phi_{\lambda s}$. 对第一级换热器 及与之相连冷屏而言, 导热路径包括对储液槽的传导 漏热 $\Phi_{\lambda 1-0}$, 对第二级换热器及冷屏的颈管导热 $\Phi_{\lambda 2-1}$ 和 杜瓦内管导热 $\Phi_{\lambda c 2-1}$; 辐射路径包括对液氦槽的辐射传 热 $\Phi_{f 1-0}$, 对第二级换热器及冷屏的辐射传热 $\Phi_{f 2-1}$. 同 理, 可对第二级换热器及与之相连冷屏进行传热分析. 实际上, 真空度高于 $1 \times 10^{-2} \mathrm{~Pa}$ 后, 残余气体传热量会减 小到低于辐射漏热量的 $5 \%$, 因此，上述模型忽略了残 余气体传导漏热.

当系统达到热平衡时，以第二级换热器及冷屏为 研究对象, 建立的热平衡式为

$$
\begin{aligned}
& \Phi_{f 3-2}+\Phi_{\lambda 3-2}+\Phi_{\lambda c 3-2}-\left(\Phi_{f 2-1}+\Phi_{\lambda 2-1}+\Phi_{\lambda c 2-1}\right) \\
& =c_{\mathrm{p}} \times \dot{m} \times \Delta T_{2},
\end{aligned}
$$

式中, $c_{\mathrm{p}}, \dot{m}, \Delta T$ 分别为冷氦气的比热、质量流量和换 
热器内的温度变化量. 同理, 针对第一级换热器及冷屏 建立热平衡式如下:

$\Phi_{f 2-1}+\Phi_{\lambda 2-1}+\Phi_{\lambda c 2-1}-\left(\Phi_{f 1-0}+\Phi_{\lambda 1-0}+\Phi_{\lambda c 1-0}\right)$

$=c_{\mathrm{p}} \times \dot{m} \times \Delta T_{1}$.

将上两式相加, 得到以第一级换热器及其冷屏与第二 级换热器及其冷屏为总体对象进行研究的热平衡关系 式为

$\Phi_{f 3-2}+\Phi_{\lambda 3-2}+\Phi_{\lambda c 3-2}-\left(\Phi_{f 1-0}+\Phi_{\lambda 1-0}+\Phi_{\lambda c 1-0}\right)$

$=c_{\mathrm{p}} \times \dot{m} \times\left(\Delta T_{1}+\Delta T_{2}\right)$.

以液氦储液槽为研究对象, 建立的热平衡式为

$\Phi_{\mathrm{tank}}=\Phi_{f 1-0}+\Phi_{\lambda 1-0}+\Phi_{\lambda c 1-0}+\Phi_{\lambda p}+\Phi_{\lambda \nu}+\Phi_{\lambda s}$.

储液槽达到热平衡状态时，储液维持储液槽低温 状态的冷量由液氦潜热 $\Phi_{\gamma}$ 提供, 即储液槽的漏热量 $\Phi_{\mathrm{tank}}=\Phi_{\gamma}$. 据此, 可计算储液槽内液氦蒸发速率和储液 槽内液氦存储时间 $t$ :

$\dot{m}=\frac{\Phi_{\mathrm{tank}}}{\gamma}, t=\frac{\rho V \gamma}{\Phi_{\mathrm{tank}}}$,

其中, $\gamma$ 为液氦汽化潜热, $\rho$ 为液氦密度, $V$ 为储液槽存储 液氦体积.

狭缝內翅式换热器在低温下对流换热系数为 ${ }^{[11]}$

$N u=0.0060 \times\left(\operatorname{Re}^{1.0239} \times \operatorname{Pr}^{-0.03367}\right)$.

当系统达到热平衡后，式(3)中对流换热冷量可由 下式计算:

$Q_{\mathrm{h}}=c_{\mathrm{p}} \times \dot{m} \times \Delta T_{1}+\Delta T_{2}=h \times A \times\left(T_{s}-T_{\text {fluid }}\right)$,

式中, $h$ 为换热器与冷氦气间对流换热系数, $A$ 为换热 器与冷氦气的接触面积, $T_{s}$ 为换热器温度, $T_{\text {fluid }}$ 为换热 器内冷氦气温度.

据此，利用上述传热模型，结合ANSYS SteadyStatic Thermal仿真模拟，对系统漏热和温度分布进行 迭代计算, 迭代计算步骤如下:

(1) 根据低温低雷诺数气体在狭缝式换热器对流 换热性能的实验研究 ${ }^{[11]}$, 假设第一级换热器温度 $T_{\mathrm{ex} 1,0}$, 由式(1) (7)计算得到储液槽漏热量 $\Phi_{\mathrm{tank}}$ 、液氦蒸发量 及各换热器内对流换热系数 $h$.

(2) 利用ANSYS Steady-Static Thermal模块通过设 置由假设计算得到的对流换热系数 $h$ 等参数模拟求解
换热器温度, 模拟过程考虑折叠冷屏接触热阻. 通过反 推得到第一级换热器温度 $T_{\mathrm{ex} 1,1}$, 将其与 $T_{\mathrm{ex} 1,0}$ 对比, 直到 满足 $\left|T_{\mathrm{ex} 1, n+1}-T_{\mathrm{ex} 1, n}\right| / T_{\mathrm{ex} 1, n}<0.1$ 时, 停止迭代, 则认为解收 玫, 最后根据上述结果重新计算系统漏热量等参数.

当换热器级数只有一级时, 通过上述迭代计算可 得到储液槽漏热量为 $0.38 \mathrm{~W}$, 换热器温度 $T_{\mathrm{ex} 1,1}$ 为 $38.25 \mathrm{~K}$, 储液槽液氦存储时间为 $25 \mathrm{~min}$, 不满足柱面 压缩实验对保温时间 $30 \mathrm{~min}$ 的要求. 当换热器级数为 两级时, 通过上述迭代计算可得到储液槽漏热量为 $0.28 \mathrm{~W}$, 第一级换热器温度为 $7.29 \mathrm{~K}$, 第二级换热器温 度为 $23.51 \mathrm{~K}$, 储液槽液氦存储时间为 $35.27 \mathrm{~min}$, 满足 保温时间的要求. 虽然随着换热器级数增多, 其冷量回 收效率会加大, 但是考虑到实验过程加工工艺, 三层及 以上结构系统较为繁杂不利于加工安装，且两级换热 器已满足实验需求, 故采用两级换热器结构设计.

采用ANSYS Steady-Static Thermal 模块对系统进 行仿真计算时, 边界条件的处理如下:

(1) 假设储液槽内壁面温度为 $4.2 \mathrm{~K}$, 加载真空罐 体外壁面温度为 $300 \mathrm{~K}$.

(2) 由于系统部件材料温度变化较大, 固体热导率 按温度插值计算, 镀金和包覆多层的表面发射率按照 0.02 设置 ${ }^{[12]}$, 表 1 为计算用到的材料热导率和发射率.

通过模拟计算样品室能够达到的最低温度, $T_{\mathrm{s}, \mathrm{top}}$ $=4.53 \mathrm{~K}, T_{\mathrm{s}, \mathrm{mid}}=4.24 \mathrm{~K}, T_{\mathrm{s}, \mathrm{bot}}=4.24 \mathrm{~K}$, 其中 $T_{\mathrm{s}, \mathrm{top}}, T_{\mathrm{s}, \text { mid }}$, $T_{\mathrm{s}, \mathrm{bot}}$ 分别为样品室温度、阀座温度、传冷座温度(图 6). 样品室的温度分布云图如图7所示. 模拟计算得到 第一级换热器温度为 $7.29 \mathrm{~K}$, 第二级换热器温度为 $23.51 \mathrm{~K}$, 储液槽漏热量 $0.28 \mathrm{~W}$, 第一级换热器对流换 热量为 $0.24 \mathrm{~W}$, 第二级换热器对流换热量为 $3.28 \mathrm{~W}$, 储 液槽存储时间为 $35.27 \mathrm{~min}$. 通过在ANSYS里面设置折 叠冷屏与圆筒形冷屏之间、各级折叠冷屏之间接触热 阻 $^{[13]}$, 求解两级折叠冷屏的温度分布, 计算得到第一级 折叠冷屏底部温度为 $201.44 \mathrm{~K}$, 第二级折叠冷屏底部 温度为 $228.03 \mathrm{~K}$.

表 1 几种材料热导率和发射率

\begin{tabular}{cccccccc}
\hline \multirow{2}{*}{ 材料 } & \multicolumn{6}{c}{ 热导率 $\left(\mathrm{W} \mathrm{m}^{-1} \mathrm{~K}^{-1}\right)$} & 发射率 \\
\cline { 2 - 7 } & $4 \mathrm{~K}$ & $20 \mathrm{~K}$ & $40 \mathrm{~K}$ & $80 \mathrm{~K}$ & $150 \mathrm{~K}$ & $300 \mathrm{~K}$ & \\
\hline 不锈钢 & 0.25 & 2 & 4.6 & 8 & 11 & 15 & 0.048 \\
紫铜 & 12000 & 11000 & 8197 & 650 & 562 & 394 & 0.020 \\
\hline
\end{tabular}




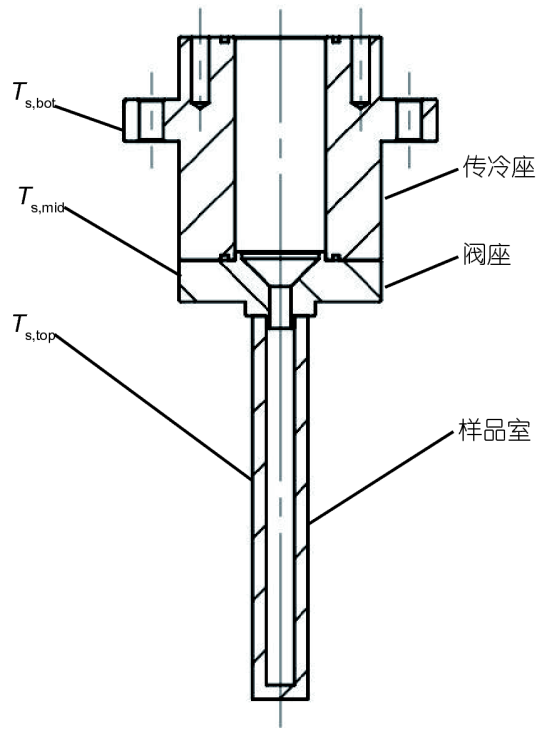

图 6 样品室温度位置

由计算结果可知, 狭缝换热器冷屏隔热形式的换 热器及冷屏温度较低(7.29 23.51 K), 这是由于换热器 内翅片插入流体中，与流体直接接触，对流换热面积 大，换热效率高，可充分回收由储液罐蒸发出来的冷 氦气的显冷. 而折叠冷屏温度较高, 主要是由于各级 折叠冷屏之间单纯靠重力压紧，两金属界面裸接产生 的接触热阻较大, 不利于冷量的传递, 导致底部折叠 冷屏温度较高, 维持在201.44 228.03 K.

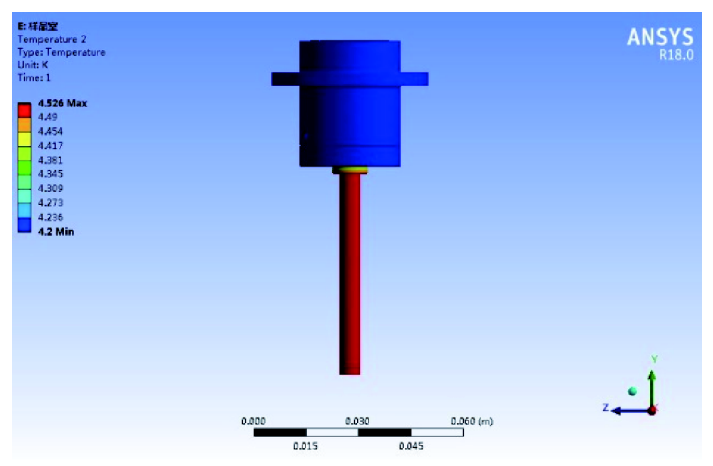

图 7 (网络版彩图)样品室温度分布云图

\section{4 实验结果}

低温靶实验系统如图8所示. 实验系统由低温靶及 其柱面加载模拟器、液氦杜瓦、样品气瓶、样品控制 罐、温度测控仪和分子洜机组等组成. 柱面加载模拟 器与液氦低温靶主体相连, 为低温靶提供真空环境. 同时，在柱面压缩实验中模拟器外部缠绕线圈为样品 室提供轴向电磁加载力. 样品气瓶、样品控制罐和真 空压力表等共同组成样品气进气系统，为样品室提供 设定的充气压力. 分子䈋对样品控制罐和柱面加载模 拟器腔体抽取真空，真空度要求高于 $1 \times 10^{-2} \mathrm{~Pa}$. 液氦 杜瓦用于向低温靶内充注液氦, 进行降温. 样品室、 阀座、传冷座上均安装二极管温度传感器, 安装位置 如图6所示.

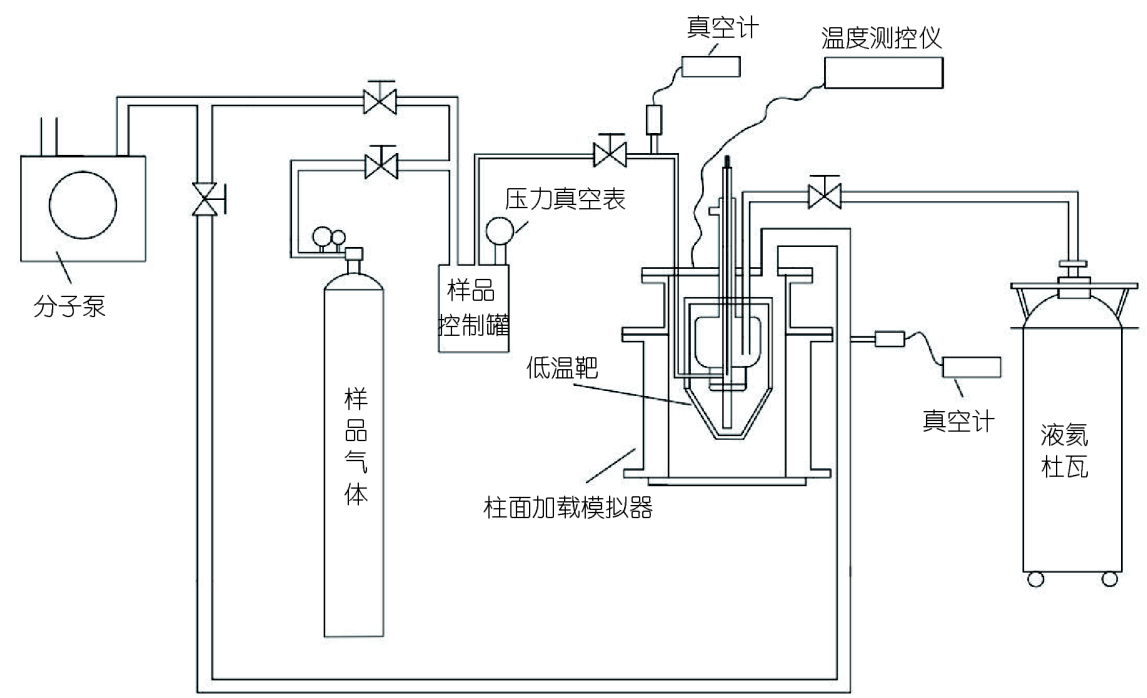

图 8 液氦低温靶实验系统示意图 
实验时, 首先利用分子原机组对低温靶主体与模 拟件之间的腔体及低温靶样品室抽真空. 待真空度高 于 $1 \times 10^{-2} \mathrm{~Pa}$ 后, 向低温靶储液槽内输入液氦对系统进 行降温. 通过装配不同形式的传冷座使样品室的温度 维持在不同的样品液化温度. 待样品室温度稳定在目 标液化温度后, 停止对样品室抽取真空, 利用阀门切 换将样品气进气系统与样品室接通, 进行样品气的充 气液化 ${ }^{[10]}$. 充气液化完成后, 需继续向储液罐输入液 氦, 将储液罐注满液氦. 然后停止输液, 关闭真空洜阀 门, 撤离液氦杜瓦和分子泵组. 此时, 低温靶所需冷量 由储液罐内液氦提供. 加载装置准备完成后, 通过控制 驱动电机使得折叠冷屏脱落, 样品室裸露于加载装置 内, 此时就能进行柱面压缩实验.

图9为低温靶样品室的降温实验曲线. 样品室最低 温度为 $4.77 \mathrm{~K}$, 由室温 $(300 \mathrm{~K})$ 降至最低温度 $(4.77 \mathrm{~K})$ 所 需时间为 $24 \mathrm{~min}$. 由图可知, 实验中样品室的降温速率 会随时间而变化, 表现为前期降温速率慢, 后期较快. 这是由于液氦刚输入系统内时, 折叠冷屏温度较高, 对 样品室辐射漏热量较大, 样品室降温速率慢, 随着折叠 冷屏温度逐渐降低, 对样品室辐射漏热量变小, 样品室 降温速率加大. 当样品室温度降至最低温后, 系统达到 热平衡, 其温度维持不变.

图10为低温靶保温实验曲线. 在停止外界液氦供 给并关闭分子柡后, 样品室温度稳定在 $4.77 \mathrm{~K}$, 波动范 围为 $\pm 0.01 \mathrm{~K}$, 维持时间为 $32 \mathrm{~min}$. 由图可知, 样品室和 阀座的温度未达到液氦温度 $4.20 \mathrm{~K}$, 主要是因为样品 室与阀座并未直接与储液槽液氦冷却面相连, 同时, 在样品室辐射热的作用下形成了温差梯度, 阀座与液

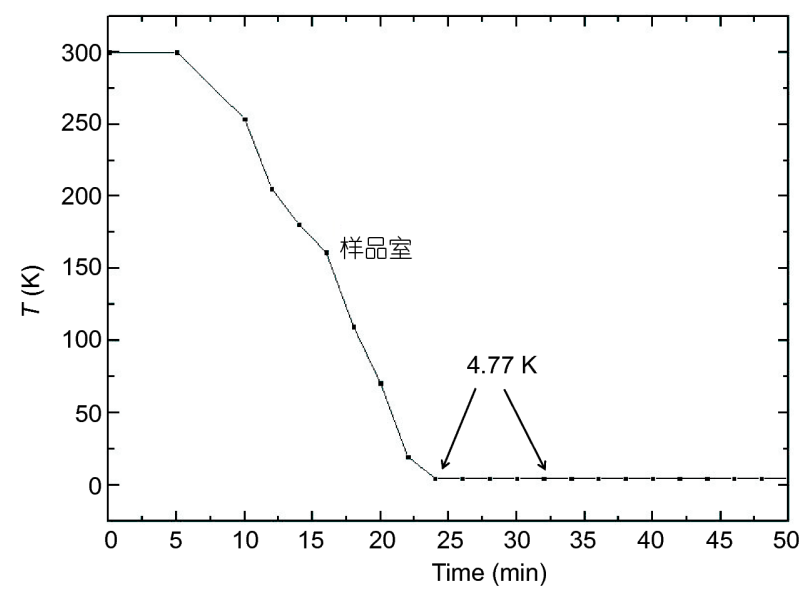

图 9 样品室降温曲线

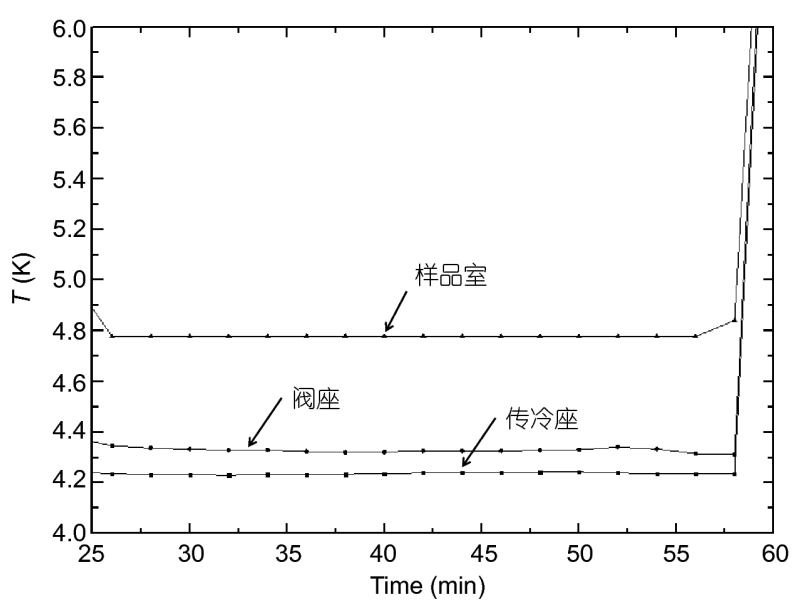

图 10 低温靶保温实验曲线

氦冷却面距离较近, 故温度较低, 而由于样品室接收 辐射漏热, 温度高于阀座温度. 保温阶段结束, 储液槽 内无液氦存储, 传冷座和样品室无制冷剂提供冷量, 此 时外界辐射热和传导热导致其温度快速升高.

实验测得各级换热器温度及冷氦气排出口气体流 量如下: 换热器 1 温度 $7.93 \mathrm{~K}$, 换热器 2 温度 $24.01 \mathrm{~K}$, 排 气口流量 $(\mathrm{LPM})$ 为 $4.11 \mathrm{~W}$, 第一级折叠冷屏底部温度 $203.56 \mathrm{~K}$, 第二级折叠冷屏底部温度 $230.14 \mathrm{~K}$, 储液槽 存储时间 $32 \mathrm{~min}$. 通过流量计测量出口气体流量 $Q_{V}$ $(\mathrm{LPM})$, 可经过式(8)计算得到液氦储液槽漏热量为

$Q_{\gamma}=\dot{m} \times \gamma=Q_{V} \times \rho \times \gamma / 60=0.29 \mathrm{~W}$.

通过测试得到的数据, 计算得到第一级换热器对流 换热量为 $0.29 \mathrm{~W}$, 第二级换热器对流换热量为 $3.50 \mathrm{~W}$. 与 模拟计算值相比, 实验测得的样品室温度比计算值高 $0.24 \mathrm{~K}$, 阀座温度高 $0.08 \mathrm{~K}$, 传冷座温度高 $0.04 \mathrm{~K}$, 实验测 得温度值和漏热量均与计算值基本一致. 分析模拟结果 与实验结果存在差异的误差原因可能是计算用材料发 射率及导热率等物性参数选取较理想.

\section{5 结论}

本文介绍了一种应用于柱面压缩实验的液氦温区 低温靶. 该低温靶通过设置两级内翅式换热器冷屏和 折叠冷屏隔热形式, 实现了液氦在有限容积内的高效 存储并维持稳定的样品室温度的目的.

用ANSYS Steady-Static Thermal 模块对低温靶的 热特性进行了仿真计算, 得到了低温靶的温度场、漏 热量和保温时间等参数. 通过实验测试, 低温靶样品 
室获得了 $4.77 \mathrm{~K}$ 的最低温度和 $\pm 0.01 \mathrm{~K}$ 的样品室温度稳 定度. 样品室由室温降至最低温所需时间为 $24 \mathrm{~min}$. 在 断开外界液氦供给和关闭真空原的情况下，样品室的 温度依然能通过储液槽液氦维持 $32 \mathrm{~min}$ 的稳定低温状
态, 实验结果与模拟结果吻合较好, 可以利用仿真结果 对低温靶系统的温度场、漏热量等进行定量分析. 理 论计算和实验测试结果表明, 该低温靶的传热结构设 计和温度特性满足柱面压缩实验的要求.

\section{参考文献}

1 Rigden S M, Ahrens T J, Stolper E M. Shock compression of molten silicate: Results for a model basaltic composition. J Geophys Res, 1988, 93: 367-382

2 Miller G H, Stolper E M, Ahrens T J. The equation of state of a molten komatiite: 1 Shock wave compression to 36 GPa. J Geophys Res, 1991 , 96: 11831

3 Collaudin B, Rando N. Cryogenics in space: A review of the missions and of the technologies. Cryogenics, 2000, 40: 797-819

4 孙承纬. 电磁加载下的高能量密度物理问题研究. 高能量密度物理, 2007, 1: 41-46

5 Nellis W J, Ree F H, van Thiel M, et al. Shock compression of liquid carbon monoxide and methane to 90 GPa (900 kbar). J Chem Phys, 1981, 75: 3055-3063

6 Lalit C C, Lee D, Ya H. High Pressure Shock Compression of Solids VII. Berlin, Heidelberg: Springer, 2005. 42-43

7 Hawke R S, Duerre D E, Huebel J G, et al. Method of isentropically compressing materials to several Megabars. J Appl Phys, 1972, 43: 27342741

8 Nellis W J, Mitchell A C. Shock compression of liquid argon, nitrogen, and oxygen to 90 GPa (900 kbar). J Chem Phys, 1980, 73: 6137-6145

9 Boriskov G V, Bykov A I, Egorov N I, et al. Isentropic compression of substances using ultra-high magnetic field: Zero isotherms of protium and deuterium in pressure range up to $\sim 5$ Mbar. Contrib Plasma Phys, 2011, 51: 339-348

10 高昶, 洪国同. 柱面压缩液氮低温靶的设计与实验研究. 真空与低温, 2014, 3: 145-149

11 Li X L, Hong G T, Wang G P. The experimental research on heat transfer coefficients of low temperature and low Reynolds number nitrogen gas. Chin Sci Bull, 2017, 62: 335-342

12 黄智斌, 朱冬梅, 唐秀风, 等. K424合金镀金膜后红外发射率变化. 稀有金属材料与工程, 2009, 38: 1402-1405

13 徐圣亚. 真空环境下低温固体界面接触热阻研究. 硕士学位论文. 北京: 中国科学院大学, 2011 


\title{
A liquid helium temperature target with two-stage internally finned cold shield heat exchanger for cylinder compression experiment
}

\author{
LI XueLiang ${ }^{1,2}$, HONG GuoTong ${ }^{1}$, WANG GuoPeng ${ }^{1,2}$, MOU Jian $^{1} \&$ LI JianGuo ${ }^{1}$ \\ ${ }^{1}$ Key Laboratory of Space Energy Conversion Technology, Technical Institute of Physics and Chemistry, Chinese Academy of Sciences, Beijing \\ 100190, China; \\ ${ }^{2}$ University of Chinese Academy of Sciences, Beijing 100049, China
}

The shock compression experiment of specimen at high-energy density state is an important way to get the state equation. The cryogenic technology is commonly used to get the high-energy density state which known as cryogenic target. This paper introduces a new kind of liquid helium cryogenic target for cylinder compression experiment. With two-stage internally finned heat exchanger cold shield and fold cold shield insulation structure, this target realizes the efficient storage of liquid helium and successfully developed the first domestic application in the liquid helium cryogenic target of cylinder compression experiment. The cavity can maintain the minimum temperature $4.77 \mathrm{~K}$ and keep stability for $32 \mathrm{~min}$ after move away the pump and helium Dewar which meet the requirement of cylinder loading experiment. This paper introduces the structure design of cryogenic target, thermal analysis with ANSYS Steady-Static Thermal module and experimental results of the target. The simulation results agree well with the experimental results.

liquid helium cryogenic target, internally finned heat exchanger cold shield, fold cold shield, numerical simulation doi: 10.1360/N092017-00359 